\title{
Prognostic value of tumor-infiltrating lymphocytes for patients with completely resected stage IIIA(N2) non-small cell lung cancer
}

\author{
Wen Feng ${ }^{1,2, *}$, Yuan $\mathrm{Li}^{3, *}$, Lei Shen ${ }^{3}$, Xu-Wei Cai ${ }^{1}$, Zheng-Fei Zhu ${ }^{2}$, Jian-Hua Chang ${ }^{4}$, \\ Jia-Qing Xiang ${ }^{5}$, Ya-Wei Zhang ${ }^{5}$, Hai-Quan Chen ${ }^{5}$, Xiao-Long Fu ${ }^{1,2}$ \\ ${ }^{1}$ Department of Radiation Oncology, Shanghai Chest Hospital, Shanghai Jiao Tong University, Shanghai, China \\ ${ }^{2}$ Department of Radiation Oncology, Fudan University Shanghai Cancer Center, Shanghai, China \\ ${ }^{3}$ Department of Pathology, Fudan University Shanghai Cancer Center, Shanghai, China \\ ${ }^{4}$ Department of Medical Oncology, Fudan University Shanghai Cancer Center, Shanghai, China \\ ${ }^{5}$ Department of Thoracic Surgery, Fudan University Shanghai Cancer Center, Shanghai, China \\ *These authors contributed equally to this work \\ Correspondence to: Xiao-Long Fu, e-mail: xlfu1964@hotmail.com
}

Keywords: lymphocytic infiltration, non-small cell lung cancer, prognosis, survival, tumor-infiltrating lymphocytes

Received: August 09, 2015

Accepted: January 01, 2016

Published: January 22, 2016

\section{ABSTRACT}

Background: The patient prognosis after complete resection for pathologic stage IIIA(N2) non-small cell lung cancer (NSCLC) remains a significant concern. The clinical relevance of the host immune response to NSCLC has yet to be established. We aimed to investigate the prognostic value of tumor-infiltrating lymphocytes (TILs) in a uniform cohort of patients with completely resected stage IIIA(N2) NSCLC.

Methods: From 2005 to 2012, consecutive patients with pathologic stage IIIA(N2) NSCLC who underwent complete resection at our institution were reviewed. For each case, full-face hematoxylin and eosin-stained sections from surgical specimens were evaluated for the TIL density. A published, recommended TIL scoring scale was followed. The patients were stratified into the TIL- or TIL+ group based on pathologic evaluation.

Results: Data from 320 patients were included in the analysis. Based on a median follow-up duration of $\mathbf{3 0 . 8}$ months, a higher density of TILs was associated with an improved postoperative survival time $(P=0.06)$. Subgroup analyses indicated that this positive effect was the greatest for patients with squamous cell carcinoma (SCC; $P=0.03$ ). Among those with SCC, the TIL+ patients experienced a significantly increased 3-year distant metastasis-free survival (DMFS) compared to the TILpatients $(60.6 \%$ versus $42.7 \%, P=0.02)$. Multivariate analyses of the 93 patients with SCC tumors confirmed that TIL+ was an independent prognostic factor for an increased DMFS ( $\mathrm{HR}=0.39,95 \% \mathrm{CI} 0.17-0.87, P=0.02)$ and a prolonged overall survival (OS; HR=0.47, 95\%CI 0.22-1.00, $P=0.05$ ).

Conclusions: Our data suggest a potential role of TILs in predicting the survival of patients with completely resected stage IIIA(N2) NSCLC. The beneficial effects of TILs were more pronounced in the prediction of the DMFS and the OS in patients with SCC. This parameter should be considered for prospective inclusion in clinical trials.

\section{INTRODUCTION}

Stage IIIA(N2) non-small cell lung cancer (NSCLC) consists a heterogeneous group of patients with distinct clinical subsets that can be classified as follows:1) stage
IIIA-1/IIIA-2, incidental mediastinal nodal involvement, found either intraoperatively in a single station or in the final pathological examination of the surgical specimen; 2) stage IIIA-3, clinical single station or multistation N2 node(s) involvement documented by computed 
tomography (CT) and/or positron emission tomography (PET)/CT imaging; and 3) stage IIIA-4, bulky or fixed cN2 involvement identified at imaging [1]. The international guidelines recommend that patients with occult-positive $\mathrm{N} 2$ nodes that are discovered at the time of pulmonary resection should continue with the planned resection along with formal mediastinal lymph node dissection and that patients with minimal N2 disease could be considered for a multimodality approach that includes surgical resection [1-3]. In our study, we evaluated patients with occult N2 identified after complete resection (IIIA-1, IIIA-2) and minimal N2 disease identified by CT or PET/CT imaging (IIIA-3). Determination of the role of surgery in a patient with minimal N2 disease was made, prior to the initiation of any therapy, by a multidisciplinary team in our institution. Complete surgical resection is still considered the initial treatment option in our country for most patients with occult or minimal N2 lesions that are determined to be resectable by a multidisciplinary team.

The patient prognosis after complete resection for pathologic stage IIIA(N2) NSCLC remains a significant concern; the 5-year overall survival (OS) rates range from $10 \%$ to $30 \%$ [4]. Postoperative chemotherapy (POCT) has a modest, but statistically significant, survival benefit for patients with resected NSCLC (absolute improvement in survival of $4 \%$ at 5 years) [5]. The postoperative radiotherapy (PORT) meta-analysis [6] described a relative increase in the risk of death with the addition of PORT for completely resected NSCLC. This detrimental effect was evident among patients who had no mediastinal involvement, whereas in patients with stage III and pN2 disease, a slight increase in survival was detected, although the difference was not statistically significant. Growing evidence suggests that PORT that is administered using the modern technique has a favorable effect on the survival of patients with completely resected $\mathrm{pN} 2$ disease [7-13]. A large multi-institutional randomized trial evaluating PORT (Lung ART) in this patient population is now under way [14]. Our group has studied the value of PORT and the PORT clinical target volume design for completely resected pathologic stage IIIA(N2) NSCLC $[15,16]$. Based on our results, patients with completely resected stage IIIA(N2) NSCLC had a 5-year OS rate of $32.8 \%$; and even after complete resection and POCT, a significant proportion of these patients developed distant metastasis $[15,16]$. These findings provided the impetus for identifying patients who are at high risk of recurrence and exploring novel therapeutic approaches for improving patient survival.

There is growing recognition that tumor progression does not simply depend upon its intrinsic behavior but rather involves a complex interaction between the tumor and host antitumor immunity $[17,18]$. The effect of the host immune response on tumors has been demonstrated by analyses of in situ immune components [19]. Accumulating data have shown that the in situ host immune response to a tumor might determine the tumor behavior or provide clinically informative prognostic biomarkers [20-24]. Evidence indicates that the type, density, and location of immune cells within tumors are better predictors of patient survival than the histopathologic methods that are currently used for the staging of colorectal cancer [24]. Promising immunotherapy approaches are under investigation, and an immunological biomarker in the tumor immune microenvironment that could be integrated into future clinical trials and translational research might eventually be identified.

In this regard, we hypothesized that the state of local immune infiltration at the time of NSCLC resection might be clinically important and measureable. Approximately two-thirds of tumor stroma inflammatory cells in NSCLC are lymphocytes; among these lymphocytes, $80 \%$ are $\mathrm{T}$ cells [25]. The ability of the cellular immune response, as evidenced by tumor-infiltrating lymphocytes (TILs), to predict survival in patients with a variety of solid tumor types has been corroborated by clinical observation [26-29]. However, the impact of the TIL patterns on the disease course of NSCLC remains to be established, and most studies are heterogeneous in terms of the disease stage (I-IV) and TIL assessment method [30-36]. As part of the pathologic workup at the University of Pittsburgh Medical Center, pathologists evaluate resected NSCLC samples for TILs $[37,38]$. These pathologists attempted to examine the relationship between the number of TILs and clinical outcome in early-stage NSCLC. Higher TIL levels were associated with improved disease-free survival among patients with resected stage I disease, but these findings did not translate into a survival benefit $[37,38]$.

In this study, we sought to investigate the prognostic significance of TILs, which were evaluated in routine histopathological sections, in a uniform cohort of patients with pathologic stage IIIA(N2) NSCLC after complete resection.

\section{RESULTS}

\section{Baseline characteristics}

Of the 375 patients who met the inclusion criteria, 18 patients were excluded because they were lost to follow-up. Tumor specimens from 357 patients were evaluated for TILs. For 37 patients, TILs could not be adequately assessed because permanent hematoxylin and eosin ( $\mathrm{H} \& \mathrm{E})$-stained sections were not available. Therefore, 320 patients were included in the analysis; their characteristics are listed in Table 1. In total, 135 $(42 \%)$ patients were categorized as TIL+; the remaining 185 patients were defined as TIL-. As shown in Table 1, no significant differences in the clinicopathological characteristics, including gender, age, smoking history, tumor differentiation, angiolymphatic invasion (ALI), number of nodes resected, number of nodes involved, and 
Table 1: Patient characteristics and predictors of the TIL density level

\begin{tabular}{|c|c|c|c|c|}
\hline Characteristics & $\begin{array}{c}\text { No. of } \\
\text { Patients }\end{array}$ & $\begin{array}{c}\text { TIL+ } \\
\text { No. }(\%)\end{array}$ & $\begin{array}{c}\text { TIL- } \\
\text { No. }(\%)\end{array}$ & Sig. ( $P$ value $)$ \\
\hline All patients & 320 & $135(42)$ & $185(58)$ & \\
\hline Gender & & & & 0.13 \\
\hline Female & 122 & $58(48)$ & $64(52)$ & \\
\hline Male & 198 & $77(39)$ & $121(61)$ & \\
\hline Age (years) & & & & 0.45 \\
\hline$\leq 60$ & 189 & $83(44)$ & $106(56)$ & \\
\hline$>60$ & 131 & $52(40)$ & $79(60)$ & \\
\hline Smoking history & & & & 0.09 \\
\hline Never/light smoker & 155 & $73(47)$ & $82(53)$ & \\
\hline Current/heavy smoker & 165 & $62(38)$ & $103(62)$ & \\
\hline Histological type & & & & 0.003 \\
\hline Adenocarcinoma & 193 & $98(51)$ & $95(49)$ & \\
\hline Squamous cell carcinoma & 93 & $28(30)$ & $65(70)$ & \\
\hline Adenosquamous carcinoma & 23 & $7(30)$ & $16(70)$ & \\
\hline Large cell carcinoma & 9 & $1(11)$ & $8(89)$ & \\
\hline Pleomorphic carcinoma & 2 & $1(50)$ & $1(50)$ & \\
\hline Histological type & & & & 0.01 \\
\hline $\mathrm{SCC}$ & 93 & $28(30)$ & $65(70)$ & \\
\hline Non-SCC & 227 & $107(47)$ & $120(53)$ & \\
\hline Tumor differentiation & & & & 0.44 \\
\hline Well & 2 & - & $2(100)$ & \\
\hline Intermediate & 155 & $64(41)$ & $91(59)$ & \\
\hline Poor & 163 & $71(44)$ & $92(56)$ & \\
\hline Angiolymphatic invasion & & & & 0.95 \\
\hline ALI- & 195 & $82(42)$ & $113(58)$ & \\
\hline ALI+ & 125 & $53(42)$ & $72(58)$ & \\
\hline No. of nodes resected & & & & 0.41 \\
\hline$\leq 20$ & 165 & $66(49)$ & $99(53)$ & \\
\hline$>20$ & 155 & $69(51)$ & $86(47)$ & \\
\hline No. of nodes involved & & & & 0.6 \\
\hline$\leq 4$ & 177 & $77(57)$ & $100(54)$ & \\
\hline$>4$ & 143 & $58(43)$ & $85(46)$ & \\
\hline Pathologic T stage & & & & 0.81 \\
\hline $\mathrm{T} 1$ & 73 & $33(24)$ & $40(22)$ & \\
\hline $\mathrm{T} 2$ & 210 & $86(64)$ & $124(67)$ & \\
\hline T3 & 37 & $16(12)$ & $21(11)$ & \\
\hline
\end{tabular}

Abbreviations: $\mathrm{TIL}=$ tumor-infiltrating lymphocyte; $\mathrm{SCC}=$ squamous cell carcinoma; $\mathrm{ALI}=$ angiolymphatic invasion.

pathologic T stage, were observed between the two groups. The designation of TIL + was significantly associated with the histological type $(P=0.01)$. The median numbers of lymph nodes that were resected and involved were 20 (range 3-67) and 4 (range 1-54), respectively, which were adopted as cut-off points. In the entire cohort $(N=320), 278(86.9 \%)$ patients received POCT, and 34 $(10.6 \%)$ patients received PORT. The use of POCT and PORT was well balanced between the two groups. Overall, $99(73 \%)$ patients in the TIL+ group and $140(76 \%)$ 
patients in the TIL- group received $\geq 4$ cycles of POCT $(P=0.63)$, and $18(13 \%)$ and $16(9 \%)$ patients in the TIL+ and TIL-groups, respectively, received PORT $(P=0.18)$.

\section{Prognostic significance of TILs}

The median follow-up duration was 26.9 months (range, 4.4-101.4 months) for all patients and 30.8 months (range, 12-101.4 months) for living patients. The locoregional recurrence-free survival (LRFS) and the distant metastasis-free survival (DMFS) were compared between the TIL + and TIL- groups. There were significant differences in the LRFS, favoring the TIL+ group (5-year LRFS $79.2 \%$ versus $63.5 \%, P=0.02$ ) (Figure 1A). However, the 5-year DMFS was $24 \%$ for the TIL+ group compared to $23.2 \%$ for the TIL- group $(P=0.3)$ (Figure 1B). For all patients, the median survival time (MST) was 42.5 months, and the 1-, 3-, and 5 -year OS rates were $90.9 \%, 54.3 \%$, and $35 \%$, respectively. The survival curves of the four-scale TIL groups are presented in Figure S1. For the patients in the TIL- and TIL+ groups, the MSTs were 35.7 and 45.5 months, respectively. The 1-, 3-, and 5-year OS rates were $88.6 \%, 49.5 \%$, and $34 \%$, respectively, in the TILgroup and $94.1 \%, 61.2 \%$, and $35.6 \%$, respectively, in the TIL+ group. The difference in the OS between the two groups trended toward significance $(P=0.06)$ (Figure 2A). In the subgroup analysis, a trend toward a longer OS was observed in all TIL+ subgroups, but significance was only reached for the subgroups of patients with squamous cell carcinoma (SCC; $P=0.03)$ and ALI $(P=0.02)$ as well as pathologic T1 stage $(P=0.03)$ (Figure $2 \mathrm{~B})$.

The results of the univariate and multivariate analyses of the relationships between the examined clinicopathological variables and the OS are shown in Table 2. In the multivariate survival analysis, male gender (HR 1.50, 95\% CI 1.04-2.15, $P=0.03$ ), poor tumor differentiation (HR 1.57, 95\%CI 1.12-2.19, $P=0.008$ ) and number of involved nodes $>4$ (HR $1.72,95 \% \mathrm{CI}$ $1.23-2.40, P=0.001$ ) were independently associated with a worse OS. Among the other covariates, the designation of TIL+ was a potential positive prognostic factor (HR 0.7, $95 \%$ CI $0.5-0.98, P=0.05$ ).

\section{Association of TILs with the histological type}

Upon further stratification of the cases based on histological type, for patients with SCC $(N=93)$, the designation of TIL + was associated with a significantly improved DMFS (3-year DMFS: $60.6 \%$ in the TIL+ group versus $42.7 \%$ in the TIL- group, $P=0.02$; Figure $3 \mathrm{~B}$ ). There were no statistically significant differences in the 3-year LRFS between the two groups, although the results regarding the LRFS showed a positive trend in the TIL+ group $(P=0.35$; Figure $3 \mathrm{~A})$. The $1-, 3-$, and 5 -year OS rates in the TIL+ group were $100 \%, 57.4 \%$, and $34.4 \%$, respectively, which were significantly higher than the respective OS rates of $84.6 \%, 38.9 \%$, and $21 \%$ in the TIL- group $(P=0.03$; Figure $3 \mathrm{C})$. The correlation between the TIL level and clinicopathological parameters for the 93 SCC tumors is listed in Table S1. No significant associations were identified between the TIL density and the patient age, gender, smoking history, tumor differentiation, ALI, pathologic T stage, or number of nodes resected and involved $(P>0.05)$. In the multivariate analysis, the designation of TIL + was a significant independent predictor of improved DMFS (HR 0.39, 95\%CI 0.17-0.87, $P=0.02$ ). Among the other clinicopathological covariates, TILs had an independent positive prognostic impact on the OS (HR $0.47,95 \% \mathrm{CI}$ $0.22-1.00, P=0.05$; Table 3).

\section{DISCUSSION}

We present a large-scale study that used routine histopathological analysis to investigate the prognostic role of TILs in this population with completely resected pathologic stage IIIA(N2) NSCLC. Overall, our cohort exhibited a better postoperative survival outcome (5-year OS, 35\%) than the corresponding historical controls [7-11]. This slightly improved survival might be secondary to more stringent patient selection because a homogeneous group of patients who received complete resection and systematic nodal assessment was selected in our study, providing a relatively uniform clinical background.

We observed a spectrum of TIL staining intensity in the surgically resected stage $\mathrm{pN} 2$ NSCLC primary tumor samples, and $42 \%$ of which were designated as TIL+ (high TIL infiltration). The proportion of TIL+ patients in our population was consistent with two other studies of early-stage NSCLC $[37,38]$ that used the same TIL scoring scale, suggesting that TIL infiltration is common in stage IIIA(N2) disease. It was shown that the proportion of TIL+ patients differed between the histological types of NSCLC as follows: TIL+ was more frequent in adenocarcinoma (ADC) patients than in SCC patients, whereas the designation of TIL + was not associated with the gender, degree of tumor differentiation, or ALI, as has been reported by others [34, 35, 38]. The association between TILs and the histological type was consistent with the observation that in stage III NSCLC, ADC was associated with the highest lymphoid index in the cancer stroma [33]. Another study on NSCLC reported a higher frequency of TIL infiltration within cancer nests in SCC compared to in $\mathrm{ADC}$, but the opposite association was demonstrated for TIL infiltration into the cancer stroma [35]. The difference in the extent of TIL infiltration between the histological types of NSCLC may be due to the heterogeneous tumor antigenicity and/or local environment of each tumor type. However, these findings should be considered and validated in future studies on TILs. 
A

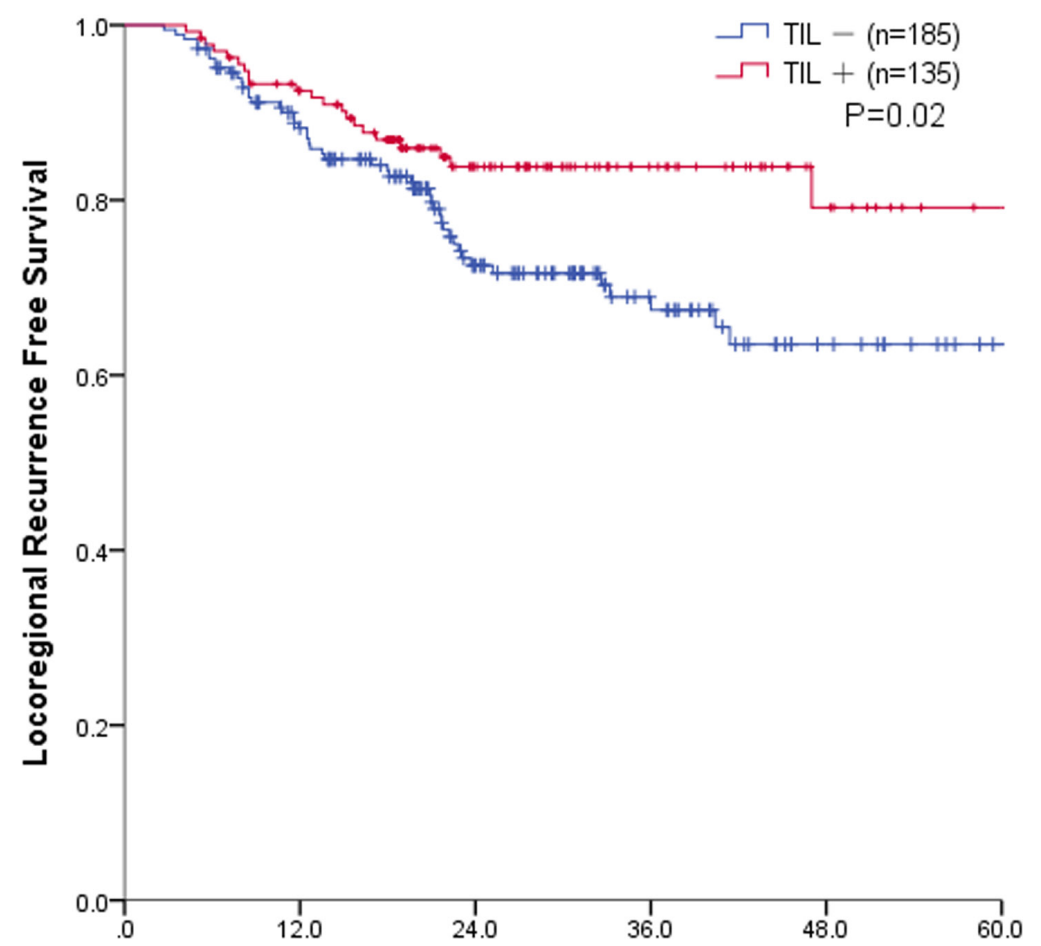

\begin{tabular}{ccccccc} 
Number at risk & \multicolumn{7}{c}{ Time (months) } \\
TIL- & 185 & 148 & 84 & 45 & 23 & 12 \\
TIL+ & 135 & 118 & 71 & 37 & 17 & 8
\end{tabular}

B

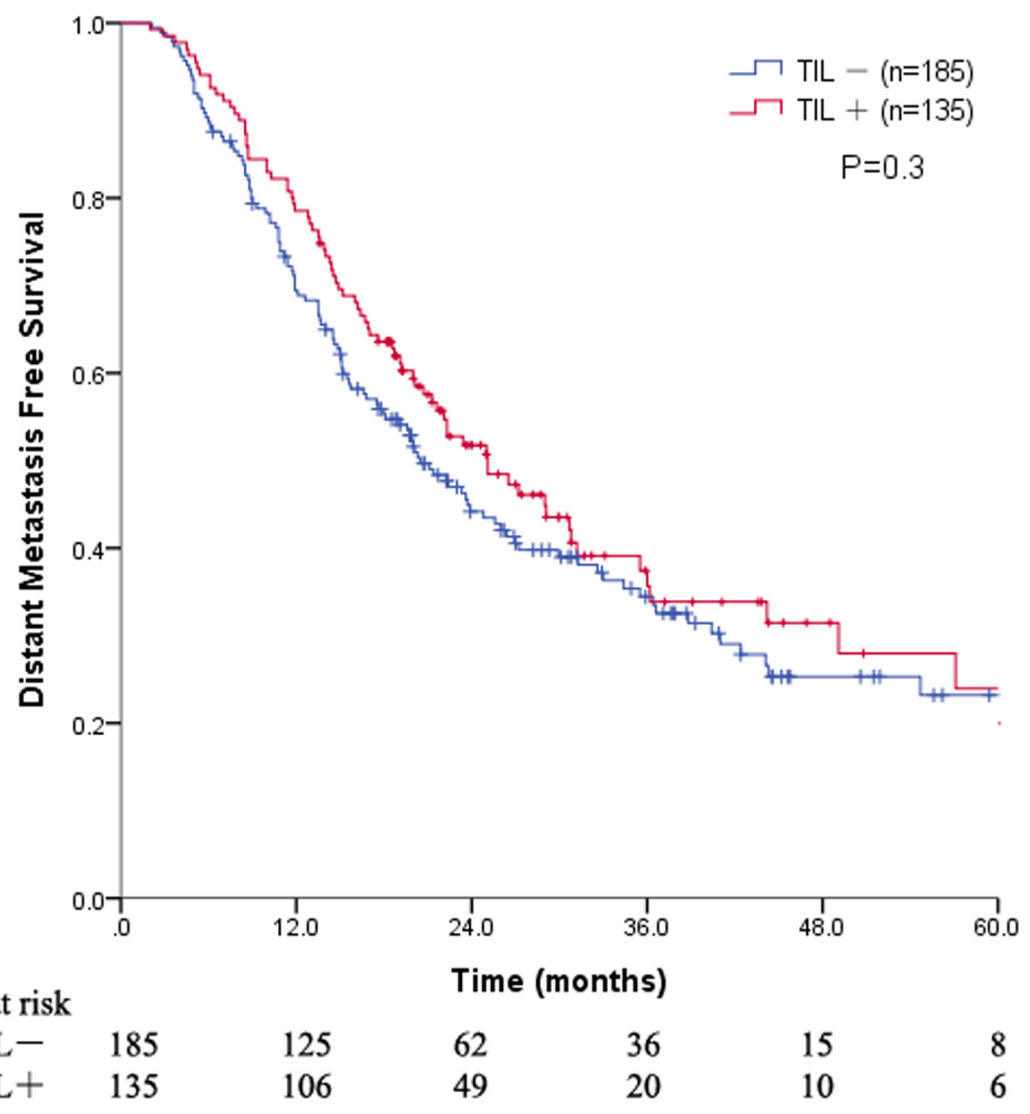

Figure 1: (A) Plot of the LRFS for all patients stratified into TIL groups; (B) Plot of the DMFS for all patients stratified into TIL groups. Abbreviations: LRFS = locoregional recurrence free survival; DMFS = distant metastasis free survival; TIL = tumor-infiltrating lymphocyte. 
A

B

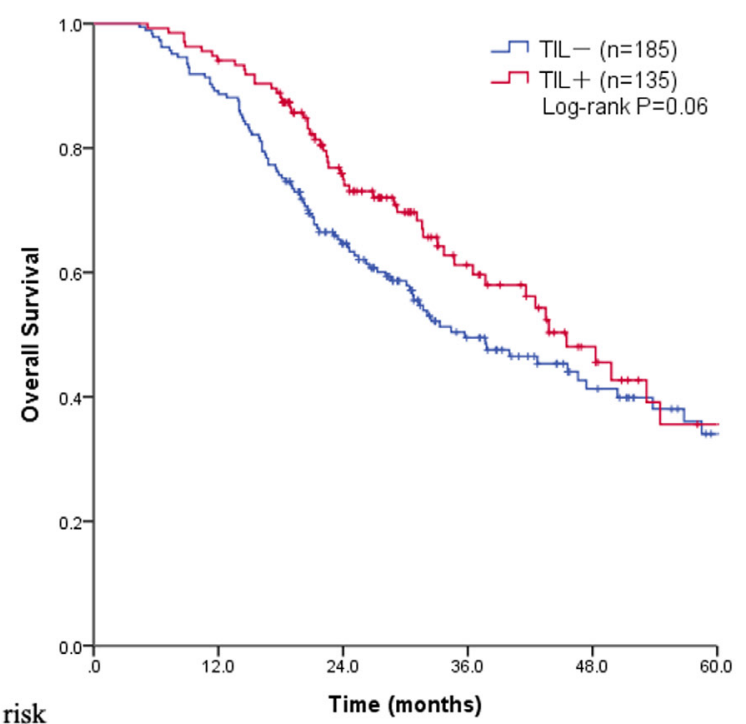

\begin{tabular}{ccccccc}
\multicolumn{2}{c}{ Number at risk } & \multicolumn{7}{c}{ Time (months) } \\
TIL- & 185 & 164 & 102 & 54 & 30 & 15 \\
TIL + & 135 & 126 & 79 & 39 & 19 & 9
\end{tabular}

Subgroup

All patients

No. of patients

HR (95\% CI) P value

Gender

Female

Male

Age (year)

$\leq 60$

$>60$

320

122

198

Smoking history

Never/light smoker

Current/heavy smoker

Histological type

Non-SCC

SCC

Tumor differentiation

Well/intermediate

Poor

ALI

ALI-

ALI+

$\mathrm{N}$ of nodes resected

$\leq 20$

$>20$

$\mathrm{N}$ of nodes involved

$\leq 4$

$>4$

Pathologic T stage

T1

T2-3

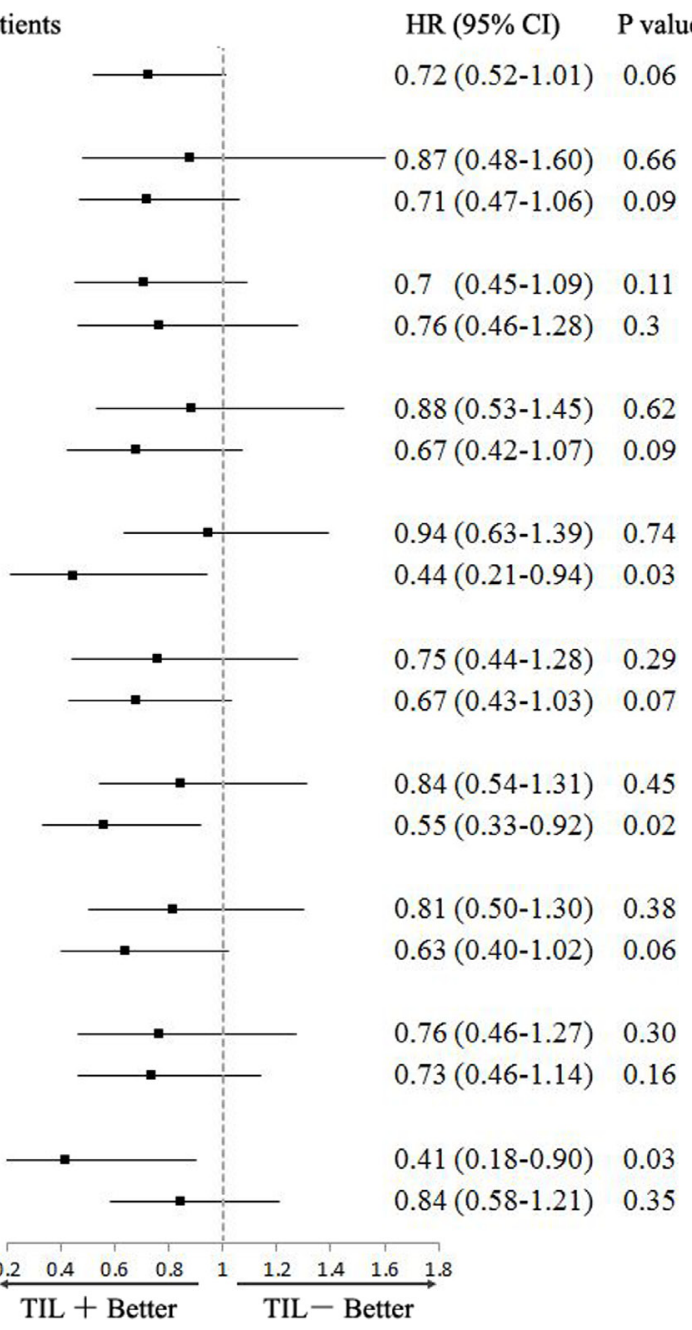

Figure 2: (A) Plot of the OS for all patients stratified into TIL groups; (B) Subgroup analyses of the OS according to each clinicopathological characteristic.Abbreviations: $\mathrm{OS}=$ overall survival; $\mathrm{TIL}=$ tumor-infiltrating lymphocyte; $\mathrm{SCC}=$ squamouscell carcinoma; $\mathrm{ALI}=$ angiolymphatic invasion; $\mathrm{CI}=$ confidence interval. 
Table 2: Univariate and multivariate analyses of the OS in all patients $(N=320)$

\begin{tabular}{|c|c|c|c|c|c|}
\hline \multirow{2}{*}{ Characteristics } & \multicolumn{3}{|c|}{ Univariable analysis } & \multicolumn{2}{|c|}{ Multivariate analysis } \\
\hline & MST (m) & 5 year $(\%)$ & $P$ value & HR $(95 \% C I)$ & $P$ value \\
\hline Gender & & & 0.001 & & 0.03 \\
\hline Female & 50.4 & $44.2 \%$ & & 1 & \\
\hline Male & 33.3 & $29.9 \%$ & & $1.50(1.04-2.15)$ & \\
\hline Age (years) & & & 0.09 & & 0.18 \\
\hline$\leq 60$ & 45.5 & $34.4 \%$ & & 1 & \\
\hline$>60$ & 31.7 & $37.2 \%$ & & $1.25(0.90-1.74)$ & \\
\hline Smoking history & & & 0.01 & & 0.95 \\
\hline Never/light smoker & 49.8 & $36.4 \%$ & & 1 & \\
\hline Current/heavy smoker & 32.5 & $33.5 \%$ & & $0.99(0.63-1.55)$ & \\
\hline Histological type & & & 0.02 & & 0.14 \\
\hline Non-SCC & 49.8 & $40.3 \%$ & & 1 & \\
\hline SCC & 33.3 & $24.0 \%$ & & $1.31(0.91-1.88)$ & \\
\hline Tumor differentiation & & & 0.001 & & 0.008 \\
\hline Well/Intermediate & 53.2 & $42.9 \%$ & & 1 & \\
\hline Poor & 31.3 & $26.7 \%$ & & $1.57(1.12-2.19)$ & \\
\hline TIL level & & & 0.06 & & 0.05 \\
\hline TIL- & 35.7 & $34.0 \%$ & & 1 & \\
\hline TIL+ & 45.5 & $35.6 \%$ & & $0.70(0.50-0.98)$ & \\
\hline ALI & & & 0.02 & & 0.11 \\
\hline ALI- & 43.8 & $42.1 \%$ & & 1 & \\
\hline ALI+ & 32.1 & $22.8 \%$ & & $1.31(0.94-1.82)$ & \\
\hline No. of nodes resected & & & 0.66 & & 0.56 \\
\hline$\leq 20$ & 41.6 & $39.2 \%$ & & 1 & \\
\hline$>20$ & 43.8 & $30.2 \%$ & & $0.91(0.65-1.26)$ & \\
\hline No. of nodes involved & & & $<0.001$ & & 0.001 \\
\hline$\leq 4$ & 49.8 & $47.5 \%$ & & 1 & \\
\hline$>4$ & 31.1 & $21.9 \%$ & & $1.72(1.23-2.40)$ & \\
\hline Pathologic T stage & & & 0.24 & & 0.83 \\
\hline $\mathrm{T} 1$ & 53.2 & $42.4 \%$ & & 1 & \\
\hline $\mathrm{T} 2$ & 41.6 & $35.1 \%$ & & $1.09(0.71-1.70)$ & \\
\hline T3 & 34.7 & $22 \%$ & & $1.20(0.67-2.16)$ & \\
\hline
\end{tabular}

Abbreviations: $\mathrm{OS}=$ overall survival; $\mathrm{MST}=$ median survival time; $\mathrm{HR}=$ hazard ratio $\mathrm{CI}=$ confidence interval; $\mathrm{TIL}=$ tumorinfiltrating lymphocyte; SCC = squamous cell carcinoma; ALI = angiolymphatic invasion.

Our data indicated that a high level of TILs in resected NSCLC specimens was associated with improved survival in patients with pathologic stage IIIA(N2) disease after complete resection, suggesting that the majority of patients with a high number of TILs in their primary tumor have had a relatively strong immune reaction against the tumor and, hence, have a better prognosis. This result was consistent with some other studies [26-29, 32, 36-38]. Accumulating data indicate that TIL infiltration in NSCLC is predominantly within the cancer stroma and that the immune response is maximal in the peritumor area $[33,36]$. In a study of stage III NSCLC, Lee at al. [33] found that the accumulation of lymphoid cells in the tumor stroma was independently associated with a favorable prognosis. Previously, two studies from the Pittsburgh Medical Center found that a higher level of TILs was associated with improved recurrencefree survival in patients with stage I NSCLC, but these findings did not translate into a significant survival benefit $[37,38]$. We reported that a higher density of TILs was 
associated with an improved LRFS $(P=0.02)$ and that there was a tendency of an improved postoperative survival time in the TIL+ group $(P=0.06)$. This finding of the prognostic significance of TIL + on local regional tumor control for patients with $\mathrm{pN} 2$ disease after complete resection has not been reported in other studies. Actually, the identification of local immunological factors (TIL+) as low-risk prognostic factors for LRF was an important clinical finding that merits and deserves further attention. The lack of a strong association between the degree of TILs and patient survival might reflect the complexity of the in situ host immune response to NSCLC. TILs are a heterogeneous group of cells with different functions and additional factors in the tumor microenvironment may regulate the functions of TILs in NSCLC [39]. Moreover, interesting results and further studies in all stages of disease seem warranted.

Given the functional heterogeneity of TILs, it is intriguing that the density of TILs assessed by simple evaluation of $\mathrm{H} \&$ E-stained primary lung tumor sections has prognostic value in pathologic stage N2 disease despite the lack of detailed information on the TIL subpopulations. These results have several important implications. First, TIL-high NSCLC may define tumors that are more immunogenic, and a study on a single immune subset may have limited value. Low or absent Treg infiltration may indicate that tumors are disregarded by the immune system, while high Treg in tumors may signal an active, but unsuccessful, attempt at tumor rejection. Second, TIL-high and TIL-low NSCLC may each reflect a distinct tumor cell biology that likely has markedly different susceptibility to immunotherapy. Finally, further evidence is needed to assess the clinical importance of the dynamics of TIL subpopulations and to establish an immune score in NSCLC that would have prognostic and predictive value in clinical decision-making.

By further stratifying the cases based on the histological type, we found that the higher OS and
DMFS associated with the TIL+ group were statistically significant in the SCC subgroup but not in the ADC subgroup. These findings suggest that the prognostic effect of TILs might differ based on the histological type of NSCLC and indicate that TILs play a unique role in SCC. Similarly, Ruffiniet al. found that the presence of TILs is significantly correlated with improved survival among SCC patients, particularly among those with stage I tumors [34]. A similar result was reported by AlShibli and associates, who indicated that stromal CD4+ and CD8+ T lymphocytes were significantly correlated with improved disease-specific survival of NSCLC and that this correlation was limited to SCC [32]. The reasons for this observation are not completely clear. To provide additional evidence supporting the relationship between TILs and histological characteristics, Yoshino and associates examined the phenotypic characteristics of TILs that were freshly isolated from various histological types of lung cancer [40]. They demonstrated differences in the phenotypic characteristics of TILs between ADC and SCC, suggesting that the type of anti-tumor immune response might be different between the histological types of NSCLC. Another finding from a recent study indicated that regulatory $\mathrm{T}$ lymphocytes (Tregs), which are known to have host immunosuppressive effects in many solid tumors, are present and significantly enriched in NSCLC patients with ADC compared to those with SCC, which partially confirms that histological characteristics are related to the TIL immune response [41]. We acknowledge that more precise and objective results would be generated if we take into account the high heterogeneity of lung adenocarcinoma and the molecular characterization of this type. Thus, our findings are preliminary, and further study is warranted to confirm them. Notably, the results, which suggest that there are different immunological characteristics between SCC and non-SCC NSCLC patients, have led to further discussions about the response of squamous and non-squamous NSCLC
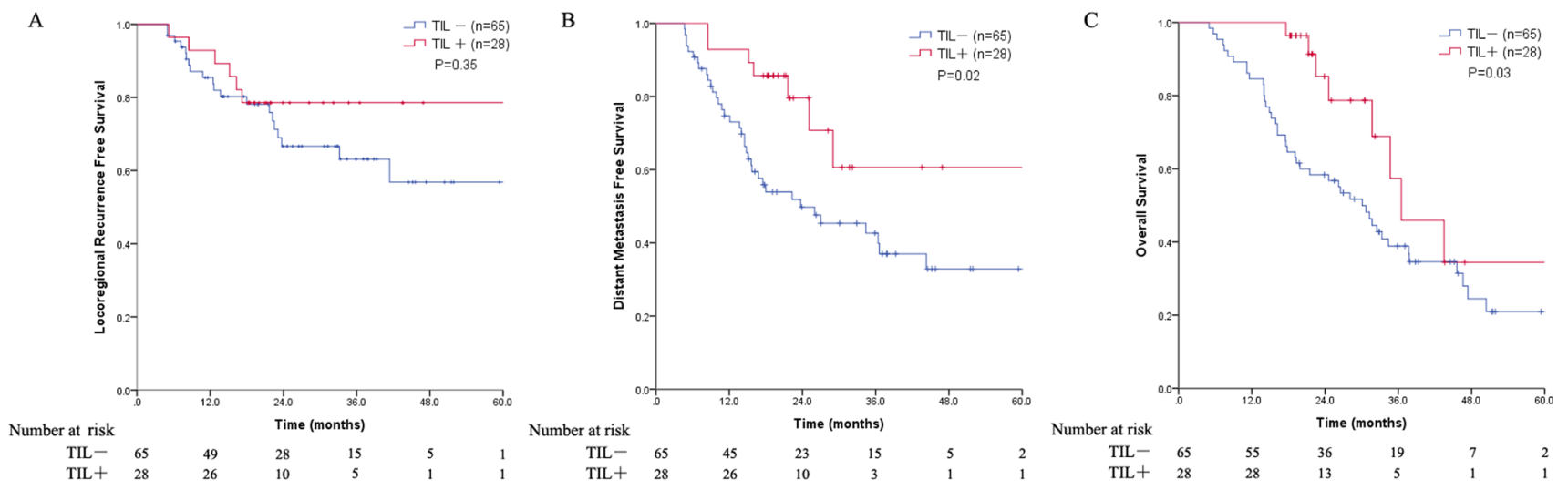

Figure 3: (A) Plot of the LRFS for patients with the SCC histological type stratified into TIL groups; (B) Plot of the DMFS for patients with the SCC histological type stratified into TIL groups; (C) Plot of the OS for patients with the SCC histological type stratified into TIL groups. Abbreviations: LRFS = locoregional recurrence free survival; DMFS = distant metastasis free survival; OS = overall survival; $\mathrm{SCC}=$ squamous cell carcinoma; TIL = tumor-infiltrating lymphocyte. 
Table 3: Univariate and multivariate analyses of the DMFS and the OS in patients with the squamous cell carcinoma (SCC) histological type $(N=93)$

\begin{tabular}{|c|c|c|c|c|c|c|c|c|c|c|}
\hline \multirow{3}{*}{ Characteristics } & \multirow{3}{*}{$\boldsymbol{N}$} & \multicolumn{4}{|c|}{ DMFS } & \multicolumn{5}{|c|}{ OS } \\
\hline & & \multicolumn{2}{|c|}{$\begin{array}{c}\text { Univariable } \\
\text { analysis }\end{array}$} & \multicolumn{2}{|c|}{ Multivariate analysis } & \multicolumn{3}{|c|}{ Univariable analysis } & \multicolumn{2}{|c|}{ Multivariate analysis } \\
\hline & & $\begin{array}{c}3 \\
\text { year } \\
(\%)\end{array}$ & $\boldsymbol{P}$ & HR $(95 \% C I)$ & $\boldsymbol{P}$ & $\begin{array}{c}3 \text { year } \\
(\%)\end{array}$ & $\begin{array}{c}5 \\
\text { year } \\
(\%)\end{array}$ & $\boldsymbol{P}$ & HR $(95 \% C I)$ & $\boldsymbol{P}$ \\
\hline Gender & & & 0.25 & & 0.23 & & & 0.11 & & 0.25 \\
\hline Female & 13 & 56.2 & & 1 & & 68.4 & 36.5 & & 1 & \\
\hline Male & 80 & 47.4 & & $1.93(0.67-5.61)$ & & 41.3 & 22.7 & & $1.73(0.67-4.47)$ & \\
\hline Age (years) & & & 0.35 & & 0.33 & & & 0.03 & & 0.05 \\
\hline$\leq 60$ & 56 & 47.5 & & 1 & & 54.9 & 23.4 & & 1 & \\
\hline$>60$ & 37 & 54.2 & & $1.37(0.73-2.56)$ & & 29.2 & 24.3 & & $1.73(1.00-3.00)$ & \\
\hline Smoking history & & & 0.41 & & 0.63 & & & 0.34 & & 0.4 \\
\hline Never/light smoker & 19 & 49.7 & & 1 & & 55.4 & 34.6 & & 1 & \\
\hline $\begin{array}{l}\text { Current/heavy } \\
\text { smoker }\end{array}$ & 74 & 48.6 & & $0.76(0.24-2.37)$ & & 42.4 & 20.4 & & $0.66(0.25-1.74)$ & \\
\hline Tumor differentiation & & & 0.47 & & 0.5 & & & 0.79 & & 0.65 \\
\hline Well/Intermediate & 45 & 47.2 & & 1 & & 49.4 & 24.3 & & 1 & \\
\hline Poor & 48 & 49 & & $0.79(0.40-1.57)$ & & 40.6 & 23.7 & & $0.86(0.45-1.65)$ & \\
\hline TIL level & & & 0.02 & & 0.02 & & & 0.03 & & 0.05 \\
\hline TIL- & 65 & 42.7 & & 1 & & 38.9 & 21.0 & & 1 & \\
\hline TIL+ & 28 & 60.6 & & $0.39(0.17-0.87)$ & & 57.4 & 34.4 & & $0.47(0.22-1.00)$ & \\
\hline ALI & & & 0.3 & & 0.27 & & & 0.3 & & 0.14 \\
\hline ALI- & 56 & 57.6 & & 1 & & 46.1 & 31.7 & & 1 & \\
\hline ALI+ & 37 & 30.2 & & $1.41(0.76-2.61)$ & & 42.8 & 9.2 & & $1.53(0.87-2.70)$ & \\
\hline No. of nodes resected & & & 0.13 & & 0.22 & & & 0.42 & & 0.17 \\
\hline$\leq 20$ & 46 & 43.1 & & 1 & & 43 & 21.1 & & 1 & \\
\hline$>20$ & 47 & 54.7 & & $0.68(0.37-1.26)$ & & 46.6 & 33.2 & & $0.66(0.36-1.20)$ & \\
\hline No. of nodes involved & & & 0.34 & & 0.21 & & & & & 0.16 \\
\hline$\leq 4$ & 62 & 57 & & 1 & & 50.4 & 27.8 & 0.16 & 1 & \\
\hline$>4$ & 31 & 31 & & $1.52(0.79-2.93)$ & & 33.3 & 13.3 & & $1.51(0.86-2.66)$ & \\
\hline Pathologic T stage & & & 0.99 & & 0.87 & & & 0.7 & & 0.7 \\
\hline $\mathrm{T} 1$ & 15 & 49.5 & & 1 & & 57.1 & 21.4 & & 1 & \\
\hline $\mathrm{T} 2$ & 59 & 53.5 & & $0.91(0.38-2.16)$ & & 41.8 & 26.6 & & $1.45(0.62-3.38)$ & \\
\hline $\mathrm{T} 3$ & 19 & 30.7 & & $1.13(0.39-3.28)$ & & 42.5 & 15.9 & & $1.34(0.48-3.73)$ & \\
\hline
\end{tabular}

Abbreviations: DMFS = distant metastasis free survival; OS = overall survival; TIL = tumor-infiltrating lymphocyte; ALI = angiolymphatic invasion; $\mathrm{HR}=$ hazard ratio; $\mathrm{CI}=$ confidence interval.

patients to immunotherapeutic agents. Recent clinical data suggest that ipilimumab, administered as first-line treatment, allows for greater improvement for patients in the squamous NSCLC subgroup than for those in the nonsquamous NSCLC subgroup [42, 43]. Indeed, additional options for combatting squamous NSCLC are urgently needed [44]. The concept that these immunological characteristics differentially predict susceptibility to immunotherapy and then help individualize adjuvant therapy in the setting of completely resected stage IIIA(N2) NSCLC will be important, clinically relevant questions to address in the near future.

It should be mentioned that our findings are preliminary and that there are limitations to this study. First, given the retrospective nature of this analysis, we cannot prove a direct causal relationship between 
a high TIL level and prolonged survival. However, this link is highly plausible for the following reason. Although the two populations were not well balanced in terms of the histological type, potential differences in the prognostic factors were corrected in the statistical comparisons. According to the multivariate analysis, the TIL density level was independently associated with an improved OS, even after adjusting for other significant clinicopathological factors, including the histological type. In the SCC subgroup, the correlations between TIL+ and other significant factors were tested, and no significant associations were identified between the density of TILs and other clinicopathological factors. In addition, multivariate analysis was performed to generate further evidence. Second, the semi-quantitative nature of the TIL scoring scale, which is considered to be easy to follow and reproducible in routine pathologic practice, represents a weakness of the present study. There are no currently established thresholds for TILs in NSCLC. The threshold for clinical decisions can be determined once a solid methodology with clinical utility is in place. Therefore, the reproducibility and reliability of this TIL scoring system need to be confirmed in a separate study before it is applied to clinical practice. Third, we focused on the degree of lymphocytic infiltration, which was assessed by the simple evaluation of $\mathrm{H} \&$ E-stained tumor sections. Given the functional heterogeneity of intratumoral lymphocytes, the analysis of TIL density as a whole might not be sufficient. Thus, further study on the immune subpopulations in the infiltrate is ongoing to establish an immune score that incorporates important immune cell subpopulations. Finally, although our preliminary findings suggested a potential prognostic role of TILs in completely resected stage IIIA(N2) disease and indicated a crucial role of TILs in SCC, they did not provide information about the predictive value of TILs for the therapeutic response. Determining whether the state of the local host immune reaction to the primary NSCLC tumor predicts the therapeutic response, especially the response to immunotherapeutic approaches, is of paramount importance for clinical management, and further investigation is warranted.

In summary, routine histopathological assessment and semi-quantitative scoring of the in situ TIL intensity provided clinically meaningful prognostic information for patients with completely resected stage IIIA(N2) NSCLC. The close examination of patients with the SCC histological type revealed that the beneficial effects of TILs were even more pronounced for predicting the DMFS and the OS. Therefore, studies assessing the outcomes and therapeutic efficacies in prospective clinical trials should consider stratifying patients based on the level of TILs.

\section{MATERIALS AND METHODS}

All consecutive patients with pathologic stage IIIA(N2) NSCLC who had undergone complete resection at our institution between January 2005 and June 2012 were retrospectively reviewed. The eligibility criteria for this study were as follows: complete resection through a surgical procedure of either lobectomy or pneumonectomy; systematic node dissection or sampling (a minimum of three N2 stations sampled or complete lymph node dissection) [2]; microscopically tumor-free resection margins; histologically proven stage pT13N2M0 NSCLC (according to the TNM classification in the UICC 7th ed. [45]); and postoperative follow-up duration $\geq 4$ months. We included patients who received POCT and/or PORT. Patients who underwent wedge resection, received neoadjuvant therapy (chemotherapy and/or radiotherapy), and/or previously presented with malignancies were excluded. Clinicopathological data, including the age, gender, smoking history, histological type, tumor differentiation, ALI, pathologic T stage, and number of lymph nodes resected and involved, were retrieved from the case history and routine pathologic reports. All pathologic characteristics were reported by qualified specialized pathologists at our institution. Smoking history was categorized as never/light exsmoker $(<100$ cigarettes in their lifetime or smoked $\leq$ 10 pack-years and stopped $\geq 15$ years ago) and current/ heavy ex-smoker. This study was approved by the institutional review board of our institution.

Permanent full-face $\mathrm{H} \& \mathrm{E}$-stained sections from surgical specimens from each case were retrieved from the pathology archives and evaluated for TILs. The evaluation of TILs was performed according to the TIL scoring scale that was previously described and published by the University of Pittsburgh Medical Center [37, 38]. The degree of lymphocyte infiltration into the tumor was scored as none (score 0 ), low (score 1), moderate (score 2), or high (score 3) (Figure 4). Low lymphocyte infiltration corresponds to the scattered presence of lymphocytes within the stroma of surrounding cancer nests; moderate lymphocyte infiltration corresponds to the modest presence of lymphocytes in the stroma without tumor nest permeation; and high lymphocyte infiltration denotes an intense or marked presence of lymphocytes in the stroma as well as the insertion of lymphocytes between tumor cells. Patients were stratified into the TIL- (none to low infiltration) or TIL+ group (moderate to high infiltration) based on pathologic evaluation. For each case, the $\mathrm{H} \&$ E-stained primary lung tumor sections were evaluated for TILs using a magnification of $\times 100$; a minimum of 5 independent fields were evaluated. Those selected fields 
were considered to be representative of the TIL density in the primary lung tumor block. For evaluating TILs, the boundaries of the invasive tumor were identified and all TILs within the tumor boundaries were scored together as stromal TILs. TILs in areas with crush artifacts, necrosis, and inflammation sites were not scored. In tumors that were heterogeneous in the TIL density in a single tumor section, we evaluated different regions and reported the average level. In cases of disagreement, all sections from the surgical specimen were reexamined to reach a consensus evaluation. Each $\mathrm{H} \&$ E-stained section was anonymized and independently scored by two expert academic pathologists (L.Y. and S.L.) who were blinded to the clinical characteristics and outcome. A total of 20 randomly selected cases from the total cohort were used to assess the intraobserver and interobserver variation. The Spearman's correlation coefficient between the two pathologists for evaluating TILs was $0.87(P<0.001)$.

The patients were generally followed every 3 months after surgery for the first 2 years and every 6-12 months thereafter. The standard follow-up evaluations included clinical assessments, chest CT scans, and ultrasound or CT of the abdomen. Treatment failure was determined by the treating physician based on the available information, including clinical assessments, imaging studies and/or pathology reports. Disease recurrence at the surgical margin, ipsilateral hilum, and/or mediastinum was considered localregional failure (LRF). All other sites of failure, including the supraclavicular zone, contralateral hilum and distant organs, were considered distant metastasis [46].

Comparisons of the categorical variables between the TIL- and TIL+ groups were performed using the Chi-square test. The OS was defined as the time from surgery to the date of death or date of the last follow-up. The LRFS was defined from the date of surgery to the date of documented LRF or the last follow-up. The DMFS was defined as the time from surgery to the date of distant metastasis or the last follow-up. The LRFS, DMFS and OS probabilities were estimated using the Kaplan-Meier method, and the significance of these results was assessed using the log-rank test. Multivariable Cox proportional hazard models (backward conditional stepwise) were used to adjust for differing risk factor distributions between the groups. Statistical analysis was performed using SPSS (version 20.0, SPSS Inc., Chicago, IL). A value of $P<0.05$ was considered statistically significant.
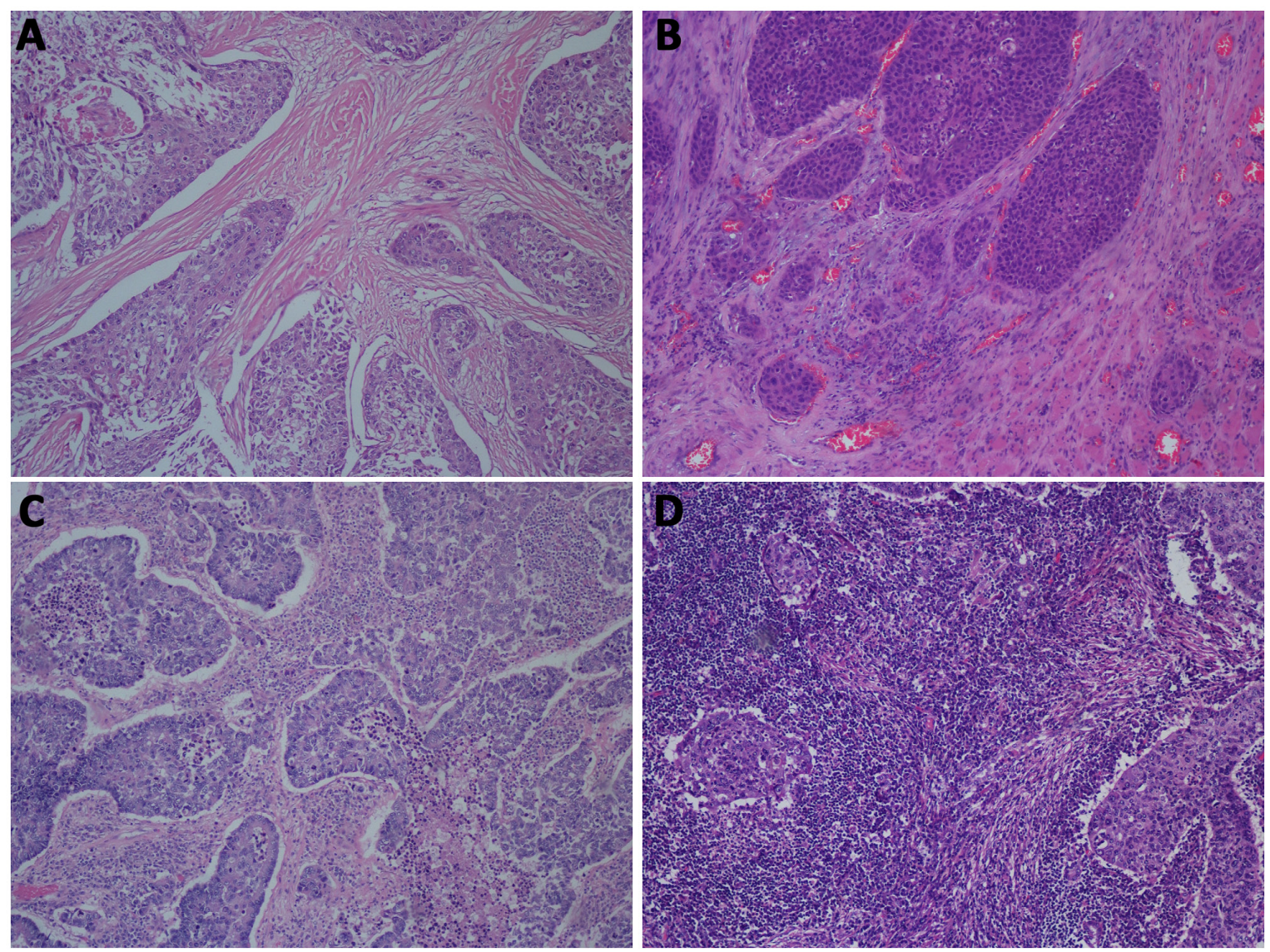

Figure 4: (A) TIL- group (score 0): almost no lymphocyte infiltration into the stroma of surrounding cancer nests; (B) TIL- group (score 1): low lymphocyte infiltration into the stroma of surrounding cancer nests; (C) TIL+ group (score 2): moderate lymphocyte infiltration into the stroma without tumor nest permeation; and (D) TIL+ group (score 3): intense lymphocyte infiltration into the stroma and insertion between tumor cells. (100X magnification, H \& E-stained sections). Abbreviations: TIL = tumor-infiltrating lymphocyte. 


\section{GRANT SUPPORT}

All authors confirm no funding supported the research included in this paper.

\section{CONFLICTS OF INTEREST}

The authors declare that they have no conflicts of interest to report.

\section{REFERENCES}

1. Eberhardt WE, De Ruysscher D, Weder W, Le Péchoux C, De Leyn P, Hoffmann H, Westeel V, Stahel R, Felip E, Peters S. Panel Members. 2nd ESMO Consensus Conference in Lung Cancer: locally advanced stage III nonsmall-cell lung cancer. Ann Oncol. 2015; 26:1573-1588.

2. National Comprehensive Cancer Network. NCCN clinical practice guidelines in oncology: non-small-cell lung cancer, v.5.2015. http://www.ncen.org.

3. Ramnath N, Dilling TJ, Harris LJ, Kim AW, Michaud GC, Balekian AA, Diekemper R, Detterbeck FC, Arenberg DA. Treatment of stage III non-small cell lung cancer: Diagnosis and management of lung cancer, 3rd ed: American College of Chest Physicians evidence-based clinical practice guidelines. Chest. 2013; 143:314S-340S.

4. Spira A, Ettinger DS. Multidisciplinary management of lung cancer. N Engl J Med. 2004; 350:379-392.

5. NSCLC Meta-analyses Collaborative Group, Arriagada R, Auperin A, Burdett S, Higgins JP, Johnson DH, Le Chevalier T, Le Pechoux C, Parmar MK, Pignon JP, Souhami RL, Stephens RJ, Stewart LA, et al. Adjuvant chemotherapy, with or without postoperative radiotherapy, in operable non-small-cell lung cancer: two meta-analyses of individual patient data. Lancet. 2010; 375:1267-1277.

6. PORT Meta-analysis Trialists Group. Postoperative radiotherapy for non-small cell lung cancer. Cochrane Database Syst Rev. 2005; 2:CD002142.

7. Lally BE, Zelterman D, Colasanto JM, Haffty BG, Detterbeck FC, Wilson LD. Postoperative radiotherapy for stage II or III non-small-cell lung cancer using the Surveillance, Epidemiology, and End Results database. J Clin Oncol. 2006; 24:2998-3006.

8. Douillard JY, Rosell R, De LenaM, Riggi M, Hurteloup P, Mahe MA. Adjuvant Navelbine International Trialist Association. Impact of postoperative radiation therapy on survival in patients with complete resection and stage I, II, or IIIA non-small-cell lung cancer treated with adjuvant chemotherapy: The Adjuvant Navelbine International Trialist Association (ANITA) randomized trial. Int J Radiat Oncol Biol Phys. 2008; 72:695-701.

9. Zou BW, Xu Y, Li T, Li W, Tang B, Zhou L, Li L, Liu Y, Zhu J, Huang M, Wang J, Ren L, Gong Y, et al. A multicenter retrospective analysis of survival outcome following postoperative chemoradiotherapy in nonsmall- cell lung cancer patients with $\mathrm{N} 2$ nodal disease. Int J Radiat Oncol Biol Phys. 2010; 77:321-328.

10. Scotti V, Meattini I, Saieva C, Agresti B, de Luca Cardillo C, Bastiani P, Livi L, Mangoni M, Di Cataldo V, Marrazzo L, Rampini A, Cipressi S, Bruni A, et al. Postoperative radiotherapy in $\mathrm{N} 2$ non-small cell lung cancer: A retrospective analysis of 175 patients. Radiother Oncol. 2010; 96:84-88.

11. Dai HH, Hui ZG, Ji W, Liang J, Lu J, Ou G, Zhou Z, Feng Q, Xiao Z, Chen D, Zhang H, Yin W, He J, et al. Postoperative radiotherapy for resected pathological stage IIIA-N2 non-small cell lung cancer: A retrospective study of 221 cases from a single institution. The Oncologist. 2011; 16:641-650.

12. Billiet C, Decaluwé H, Peeters S, Vansteenkiste J, Dooms C, Haustermans K, De Leyn P, De Ruysscher D. Modern postoperative radiotherapy for stage III non-small cell lung cancer may improve local control and survival: a metaanalysis. Radiother Oncol. 2014; 110:3-8.

13. Robinson CG, Patel AP, Bradley JD, DeWees T, Waqar SN, Morgensztern D, Baggstrom MQ, Govindan R, Bell JM, Guthrie TJ, Colditz GA, Crabtree TD, Kreisel D, et al. Postoperative radiotherapy for pathologic $\mathrm{N} 2$ non-small-cell lung cancer treated with adjuvant chemotherapy: a review of the National Cancer Data Base. J Clin Oncol. 2015; 33:870-876.

14. Le Péchoux C. Role of postoperative radiotherapy in resected non-small cell lung cancer: a reassessment based on new data. Oncologist. 2011; 16:672-681.

15. Feng W, Zhang Q, Fu XL, Cai XW, Zhu ZF, Yang HJ, Xiang JQ, Zhang YW, Chen HQ. The emerging outcome of postoperative radiotherapy for stage IIIA(N2) non-small cell lung cancer patients: based on the three-dimensional conformal radiotherapy technique and institutional standard clinical target volume. BMC Cancer. 2015; 15:348.

16. Feng W, Fu XL, Cai XW, Yang HJ, Wu KL, Fan M, Xiang JQ, Zhang YW, Chen HQ. Patterns of local-Regional failure in completely resected stage IIIA(N2) non-small cell lung cancer cases: implications for postoperative radiation therapy clinical target volume design. Int J Radiat Oncol Biol Phys. 2014; 88:1100-1107.

17. Hanahan D, Weinberg RA. Hallmarks of cancer: the next generation. Cell. 2011; 144:646-674.

18. Fridman WH, Pagès F, Sautès-Fridman C, Galon J. The immune contexture in human tumours: impact on clinical outcome. Nat Rev Cancer. 2012; 12:298-306.

19. Ogino S, Galon J, Fuchs CS, Dranoff G. Cancer immunologyanalysis of host and tumor factors for personalized medicine. Nat Rev Clin Oncol. 2011; 8:711-719.

20. Mlecnik B, Tosolini M, Kirilovsky A, Berger A, Bindea G, Meatchi T, Bruneval P, Trajanoski Z, Fridman WH, Pagès F, Galon J. Histopathologic-based prognostic factors of colorectal cancers are associated with the state of the local immune reaction. J Clin Oncol. 2011; 29:610-618. 
21. Pagès F, Galon J, Dieu-Nosjean MC, Tartour E, SautèsFridman C, Fridman WH. Immune infiltration in human tumors: a prognostic factor that should not be ignored. Oncogene. 2010; 29:1093-1102.

22. Loi S, MacCallum P. Host antitumor immunity plays a role in the survival of patients with newly diagnosed triplenegative breast cancer. J Clin Oncol. 2014; 32:2936-2938.

23. Galon J, Mlecnik B, Bindea G, Angell HK, Berger A, Lagorce C, Lugli A, Zlobec I, Hartmann A, Bifulco C, Nagtegaal ID, Palmqvist R, Masucci GV, et al. Towards the introduction of the ,Immunoscore' in the classification of malignant tumours. J Pathol. 2014; 232:199-209.

24. Galon J, Costes A, Sanchez-Cabo F, Kirilovsky A, Mlecnik B, Lagorce-Pagès C, Tosolini M, Camus M, Berger A, Wind $\mathrm{P}$, Zinzindohoué F, Bruneval $\mathrm{P}$, Cugnenc $\mathrm{PH}$, et al. Type, density, and location of immune cells within human colorectal tumors predict clinical outcome. Science. 2006; 313:1960-1964.

25. Kataki A, Scheid P, Piet M, Marie B, Martinet N, Martinet Y, Vignaud JM. Tumor infiltrating lymphocytes and macrophages have a potential dual role in lung cancer by supporting both host-defense and tumor progression. J Lab Clin Med. 2002; 140:320-328.

26. Gooden MJ, de Bock GH, Leffers N, Daemen T, Nijman HW. The prognostic influence of tumour-infiltrating lymphocytes in cancer: a systematic review with meta-analysis. $\mathrm{Br} \mathrm{J}$ Cancer. 2011; 105:93-103.

27. Thomas NE, Busam KJ, From L, Kricker A, Armstrong BK, Anton-Culver H, Gruber SB, Gallagher RP, Zanetti R, Rosso S, Dwyer T, Venn A, Kanetsky PA, et al. Tumorinfiltrating lymphocyte grade in primary melanomas is independently associated with melanoma-specific survival in the population-based genes, environment and melanoma study. J Clin Oncol. 2013; 31:4252-4259.

28. Adams S, Gray RJ, Demaria S, Goldstein L, Perez EA, Shulman LN, Martino S, Wang M, Jones VE, Saphner TJ, Wolff AC, Wood WC, Davidson NE, et al. Prognostic Value of Tumor-Infiltrating Lymphocytes in Triple-Negative Breast Cancers From Two Phase III Randomized Adjuvant Breast Cancer Trials: ECOG 2197 and ECOG 1199. J Clin Oncol.2014; 32:2959-2967.

29. Jamieson NB, Mohamed M, Oien KA, Foulis AK, Dickson EJ, Imrie CW, Carter CR, McKay CJ, McMillan DC. The relationship between tumor inflammatory cell infiltrate and outcome in patients with pancreatic ductal adenocarcinoma. Ann Surg Oncol. 2012; 19:3581-3590.

30. Bremnes RM, Al-Shibli K, Donnem T. The role of tumorinfiltrating immune cells and chronic inflammation at the tumor site on cancer development, progression, and prognosis: emphasis on non-small cell lung cancer. J Thorac Oncol. 2011; 6:824-833.

31. Suzuki K, Kachala SS, Kadota K, Shen R, Mo Q, Beer DG, Rusch VW, Travis WD, Adusumilli PS. Prognostic immune markers in non-small cell lung cancer. Clin Cancer Res. 2011; 17:5247-5256.
32. Al-Shibli KI, Donnem T, Al-Saad S, Persson M, Bremnes RM, Busund LT. Prognostic effect of epithelial and stromal lymphocyte infiltration in non-small cell lung cancer. Clin Cancer Res.2008; 14:5220-5227.

33. Lee TK, Horner RD, Silverman JF, Chen YH, Jenny C, Scarantino CW. Morphometric and morphologic evaluations in stage III non-small cell lung cancers: prognostic significance of quantitative assessment of infiltrating lymphoid cells. Cancer. 1989; 63:309-316.

34. Ruffini E, Asioli S, Filosso PL, Lyberis P, Bruna MC, Macrì L, Daniele L, Oliaro A. Clinical significance of tumor-infiltrating lymphocytes in lung neoplasms. Ann Thorac Surg. 2009; 87:365-371.

35. Mori M, Ohtani H, Naito Y, Sagawa M, Sato M, Fujimura S, Nagura H. Infiltration of CD8+ T cells in non-small cell lung cancer is associated with dedifferentiation of cancer cells, but not with prognosis. Tohoku J Exp Med. 2000; 191:113-118.

36. Hiraoka K, Miyamoto M, Cho Y, Suzuoki M, Oshikiri T, Nakakubo Y, Itoh T, Ohbuchi $\mathrm{T}$, Kondo $\mathrm{S}$, Katoh $\mathrm{H}$. Concurrent infiltration by CD8+ T cells and CD4+ T cells is a favorable prognostic factor in non-small-cell lung carcinoma. Br J Cancer. 2006; 94:275-280.

37. Kilic A, Landreneau RJ, Luketich JD, Pennathur A, Schuchert MJ. Density of tumor-infiltrating lymphocytes correlates with disease recurrence and survival in patients with large non-small-cell lung cancer tumors. J Surg Res. 2011; 167:207-210.

38. Horne ZD, Jack R, Gray ZT, Siegfried JM, Wilson DO, Yousem SA, Nason KS, Landreneau RJ, Luketich JD, Schuchert MJ. Increased levels of tumor-infiltrating lymphocytes are associated with improved recurrence-free survival in stage 1A non-small-cell lung cancer. J Surg Res. 2011; 171:1-5.

39. Sasada T, Suekane S. Variation of tumor-infiltrating lymphocytes in human cancers: controversy on clinical significance. Immunotherapy. 2011; 3:1235-1251.

40. Yoshino I, Yano T, Murata M, Miyamoto M, Ishida T, Sugimachi K, Kimura G, Nomoto K. Phenotypes of lymphocytes infiltrating non-small cell lung cancer tissues and its variation with histological types of cancer. Lung Cancer. 1993; 10:13-19.

41. Black CC, Turk MJ, Dragnev K, Rigas JR. Adenocarcinoma contains more immune tolerance regulatory t-cell lymphocytes (versus squamous carcinoma) in non-small-cell lung cancer. Lung. 2013; 191:265-270.

42. Zielinski C, Knapp S, Mascaux C, Hirsch F. Rationale for targeting the immune system through checkpoint molecule blockade in the treatment of non-small-cell lung cancer. Ann Oncol. 2013; 24:1170-1179.

43. Lynch TJ, Bondarenko I, Luft A, Serwatowski P, Barlesi F, Chacko R, Sebastian M, Neal J, Lu H, Cuillerot JM, Reck M. Ipilimumab in combination with paclitaxel and carboplatin as first-line treatment in stage IIIB/IV 
non-small-cell lung cancer: results from a randomized, double-blind, multicenter phase II study. J Clin Oncol. 2012; 30:2046-2054.

44. Stinchcombe TE. Unmet needs in squamous cell carcinoma of the lung: potential role for immunotherapy. Med Oncol. 2014; 31:960.
45. Detterbeck FC, Boffa DJ, Tanoue LT. The new lung cancer staging system. Chest. 2009; 136:260-271.

46. Higgins KA, Chino JP, Berry M, Ready N, Boyd J, Yoo DS, Kelsey CR. Local failure in resected N1 lung cancer: implications for adjuvant therapy. Int J Radiat Oncol Biol Phys. 2012; 83:727-733. 